\title{
Mujer ciudadana, universitaria y terapeuta ocupacional. Contrastes contextuales de los años sesentas y setentas desde la Universidad del Valle y la Universidad Nacional de Colombia
}

\author{
Citizen, university student and occupational therapist woman. \\ Contextual contrast in the sixties to seventies from the \\ Universidad del Valle and the \\ Universidad Nacional de Colombia
}

\author{
Lizeth Lorena Gómez Valencia ${ }^{1}$ \\ Daniela Vanessa Palma Arroyo ${ }^{2}$ \\ Laura Vanessa Rodríguez Mendoza ${ }^{3}$
}

Recibido: 28 de octubre 2016 • Enviado para modificación: 4 de diciembre 2016 • Aceptado: 15 de enero 2017

Gómez, L., Palma, D., Rodríguez, L. (2016). Mujer ciudadana, universitaria y terapeuta ocupacional. Contrastes contextuales de los años sesentas y setentas desde la Universidad del Valle y la Universidad Nacional de Colombia. Revista Ocupación Humana, 16 (2), 81-92.

Resumen: La configuración de la Terapia Ocupacional en Colombia como una profesión constituida para mujeres es uno de los hallazgos más significativos de dos trabajos de profundización e investigación que motivan este análisis, orientado a describir la conformación del rol femenino en la Terapia Ocupacional dentro del contexto político, social, económico y educativo colombiano en las décadas de los sesentas y setentas del siglo XX. Se trata de dos investigaciones realizadas en lugares del país distintos (Santiago de Cali y Bogotá), ambas emplearon la metodología de investigación histórica en el marco del macroproyecto Historias de la Terapias Ocupacionales en Colombia. Con base en el análisis crítico de hechos y eventos, los dos proyectos convergen al encontrar la relación entre la construcción social de la mujer, como ser dedicado al cuidado de otros, y sus opciones de participación en esferas políticas y educativas, lo que resulta clave para la historia de la profesión y su trayectoria en el país.

Palabras clave: Terapia Ocupacional, educación de la mujer, participación de la mujer, investigación histórica

\footnotetext{
Abstract: The configuration of Occupational Therapy in Colombia, as a profession created for women, is one of the most significant findings that has encouraged publishing the results of two investigational products, with the aim of describing the formation of women's role in Occupational Therapy within the Colombian political, social, economic and educational

${ }^{1}$ Terapeuta Ocupacional. Earlsdon Primary School. Coventry, Reino Unido. lizgova05@gmail.com

2 Terapeuta Ocupacional. Vicerrectoría de Bienestar Universitario, Universidad del Valle. Cali, Colombia. danielapalmaarroyo@correounivalle.edu.co

${ }^{3}$ Terapeuta Ocupacional. Estudiante Maestría en Neurociencias, Universidad Nacional de Colombia. Laboratorio desarrollo, juego, aprendizaje y lúdica, Departamento de la Ocupación Humana, Universidad Nacional de Colombia. Bogotá, Colombia. Ivrodriguezm@unal.edu.co
} 
context during the 1960s and 1970s in the 20th century. This article is about two projects conducted in two different regions of the country (Santiago de Cali and Bogotá). Both used the historical research methodology within the project Historias de las Terapias Ocupacionales en Colombia. According to a thorough analysis of the facts and events, both investigation projects converge by finding a relationship between the social construction of the woman as a care giver, and her options to participate in political and educational fields. This relation is key in the history of the profession and its trajectory.

Key words: Occupational Therapy, women's education, women's participation, historical research.

\section{Introducción}

Si educar a un hijo es hacer de él un hombre, la educadora, (en tal caso la

madre) necesita conocer su misión, necesita de cultura, autoridad, salud, responsabilidad, libertad y fortaleza [...] Nadie niega que la mujer nació para la maternidad, para el hogar, para la familia [...] Pero icómo se desempeña su altísima misión cuando ignora los deberes y derechos que le corresponden? Es preciso, pues, que la mujer se eduque.

(Correa, 1947, p. 128).

En el marco del macroproyecto Historias de las Terapias Ocupacionales en Colombia, diferentes universidades del país han participado de la construcción del contexto de formación de la profesión, entre ellas, la Universidad del Valle, sede Santiago de Cali, y la Universidad Nacional de Colombia, sede Bogotá.

En Cali se desarrolló, como proyecto de profundización, la identificación de los momentos de la historia, las prácticas y los discursos de la Terapia Ocupacional en esa ciudad en los años setenta (Palma \& Gómez, 2015). Ello se realizó a través de la conformación de un archivo histórico y considerando como ideas centrales la construcción de la historia como genealogía (Melgarejo, 2000), carácter particular del pensar histórico que permite cuestionarse respecto a cómo se ha llegado a ser lo que se es, y la memoria colectiva, como saber de la experiencia de un grupo de expertos, la forma como conciben su pasado, como construyen el presente y planean su futuro.

Por su parte en la ciudad de Bogotá, el grupo de la Universidad Nacional realizó su investigación bajo la modalidad de trabajo de grado, el cual describió los inicios y la trayectoria del programa de Terapia Ocupacional en los años 1966 a 1989. Se centró en la comprensión, reconocimiento, relación y reconstrucción de sucesos encontrados en documentos y relatos de actores relevantes en la historia de la Terapia Ocupacional de la Universidad Nacional (Escobar \& Rodríguez, 2015).

La investigación de Escobar y Rodríguez (2015) permitió develar que el primer programa de formación en Terapia Ocupacional del país emerge en la Universidad Nacional de Colombia durante los años sesenta, tras identificarse la necesidad de formación de personal en el pabellón de rehabilita- 
ción del Hospital San Juan de Dios de Bogotá, y que la profesión se identificó como femenina. Por su parte, aunque en la Universidad del Valle aún no se iniciaba la institucionalización de la Terapia Ocupacional, existen evidencias de la participación de la mujer dentro del Hospital Universitario del Valle como voluntaria para el cuidado de enfermos por poliomielitis, y posteriormente liderando prácticas de Terapia Ocupacional dentro de los servicios de Medicina Física y Rehabilitación en el Instituto Colombiano de los Seguros Sociales.

Motivadas por los hallazgos en torno al género y la configuración de la Terapia Ocupacional, el presente artículo propone un diálogo entre los eventos encontrados en las dos investigaciones desde una perspectiva interpretativa de los mismos. Se pretende así describir y reflexionar en torno al contexto histórico en el que transcurre la creación de los programas de Terapia Ocupacional en la Universidad Nacional y la Universidad del Valle, así como al proceso de vinculación de las mujeres a la vida educativa, política y social.

\section{Mujeres y educación: contrastes contextuales}

Las luchas y construcciones de la mujer alrededor de su identidad se han ubicado desde los años treinta del siglo XX, cuando, según Cohen (2001), "el movimiento emancipatorio que se inició en 1930 impugnó las bases sobre las cuales se sostenía la posición de la mujer, dando lugar a tres tipos de reivindicaciones: el manejo de los bienes por la mujer casada, acceso a ma- yor nivel de educación y el derecho al sufragio" (p.130). Estas se concretaron en la Ley 28 de 1932 sobre reformas civiles y régimen patrimonial en el matrimonio; un gran avance para la vida de la mujer de la época que más adelante daría lugar a fuertes debates sobre la remuneración que debían recibir por trabajar (en el momento era alrededor de la mitad del sueldo de un hombre), el tipo de empleo (generalmente de forma ocasional, en fábricas contratadas como "mano de obra no especializada"), la vinculación a las universidades y el tipo de educación que debían recibir. Aun así, ya existían mujeres profesionales en Colombia, quienes habían tenido la posibilidad de formarse en el extranjero y regresaban para ocupar altos cargos, apalancadas por las facilidades económicas de sus familias.

De otra parte, las configuraciones de la estructura social en el ámbito de la educación alimentaron el deseo urgente de reformar la educación femenina, ello implicaba el acceso al diploma de bachiller, que incrementaría la matrícula universitaria. Fue a partir de 1932 que en Colombia se dio apertura para que las mujeres de la élite ingresaran a la universidad, a carreras consideradas aptas para ellas $y$, en gran parte, sin necesidad de tener título de bachiller. Posteriormente, "en 1933 el Decreto 227 hizo extensiva la reforma de la enseñanza primaria y secundaria a los establecimientos de educación femenina y posibilitó la emisión de diplomas de bachiller" (Herrera, 1993, p. 13). Sin embargo, la educación para la mujer se dirigió a inculcar conocimientos relacionados 
con su condición de madre y esposa, a buscar la formación de una mujer moderna, preocupada por ser útil a la sociedad, pero evitando el conflicto entre sus aspiraciones profesionales y las funciones tradicionales que por naturaleza de género le habían sido concedidas.

Siendo el bachillerato un requisito para la admisión a estudios universitarios, en esta época la vinculación de las mujeres a la universidad era mínima, quienes accedían lo hacían a programas propios del cuidado y la educación de otros. Así, Cohen (2001) refiere que quienes emprendieron la lucha por su acceso a la educación superior se habían graduado como educadoras de escuelas normales, eran egresadas de institutos acreditados para ofrecer cursos prácticos de comercio, artes mecánicas y cuidado de la salud, ejercían como directoras y maestras en las escuelas femeninas de artes y oficios o eran estudiantes de colegios públicos y privados. Ya se evidenciaba un gran cambio en los proyectos de vida de las mujeres colombianas.

Según Herrera (1993), en 1943 la mujer representaba el $43 \%$ de la población estudiantil; si bien en términos generales este no parece un porcentaje bajo, es necesario matizarlo a medida que se asciende en los niveles educativos, especialmente en la educación superior, donde solo representaba el 2\% de los 5.113 estudiantes inscritos. No cabe duda que la ampliación de la cobertura en la educación femenina, y con ella el acceso de la mujer a la universidad, constituye uno de los hechos más importantes de la época, pues al finalizar la República Liberal ${ }^{4}$ cerca de medio millón de mujeres recibían educación. Este avance debe relacionarse con los logros alcanzados en el terreno de los derechos civiles y políticos, que fueron consagrando en leyes los forcejeos de la mujer por acceder a los derechos ciudadanos en pie de igualdad con el hombre.

Los discursos que justificaban el acceso de la mujer colombiana a la formación universitaria se empeñaban en establecer que las áreas de conocimiento permitidas para ellas concordaran con los roles en los cuales eran competentes, siendo el objetivo preciso el de fortalecer dichos conocimientos para desempeñarse de manera más idónea en el lugar de la sociedad al que pertenecían. El siguiente enunciado, encontrado en uno de los números de la revista Letras y Encajes de 1947, hace alusión a esta afirmación:

Es preciso pues que la mujer se eduque. Que vaya a las universidades no para escalar las montañas en busca de minas, ni para estudiar el manejo de las fuerzas que subyugan la naturale$\mathrm{za}$, ni otras muchas profesiones que no están de acuerdo con su idiosincrasia pero que estudie todo aquello que tenga por meta elevar la condición moral de la mujer, la condición de la familia y el mejoramiento de los pueblos (...) que luego con un gran

\footnotetext{
${ }^{4}$ Se entiende la Republica Liberal como el periodo histórico comprendido entre 1934 y 1938, bajo el gobierno de Alfonso López Pumarejo, durante el cual se llevó a cabo la reforma constitucional de 1936, que implicó nuevas exigencias económicas, políticas y sociales producidas por las transformaciones coyunturales de 1930, cuando se puso fin a casi medio siglo de gobiernos conservadores.
} 
caudal de ciencia y una gran dosis de responsabilidad, vaya a engrandecer el hogar ya formado y a mantener encendido, no el fuego material, sino aquel que la mano omnipotente colocó en sus entrañas y que solo manos de mujer pueden mantener encendido. (Correa, 1947, p. 118).

Para ese mismo año fueron incluidos cursos de economía doméstica en el ámbito de la enseñanza universitaria para las mujeres, entendidos no solamente como el aprendizaje de las labores domésticas tradicionales, sino como un programa científico y de mayor complejidad para mejorar la vida familiar, y por su medio, a la comunidad (López, 2002). Se destacaba que las carreras femeninas debían ser aquellas relacionadas con la asistencia social, la enfermería, la higiene y la educación, entre otras.

La construcción social de la mujer, y lo femenino, continuó hasta marcar un nuevo hito, conferido en el Acto Legislativo № 3 de 1954, que otorgó a las colombianas el derecho a sufragar (Aguilera, 2003). Este hecho generó un cambio en la perspectiva femenina, pues con él las mujeres serían tomadas en cuenta como ciudadanas aptas para tomar decisiones, no sólo para elegir, también para ser elegidas: "se le dio así la posibilidad de intervenir en la dirección y manejo de los destinos del país y se ampliaron las posibilidades de participar en la vida laboral, cultural y científica de la Nación" (Subgerencia Cultural del Banco de la República, 2015).

La Universidad Nacional de Colombia, en el ambiente de renovación del gobierno liberal de Alfonso López Pumarejo, abrió sus puertas por primera vez en Colombia a las jóvenes que aspiraban a cursar una carrera diferente al proyecto matrimonial y familiar al cual habían estado adscritas de manera exclusiva. La entrada de las mujeres a la Universidad Nacional fue masivo al llegar los años sesentas; según Munevar (2015), anteriormente ingresaba una, dos, máximo tres, en los sesentas esto cambió, ingresaban seis, siete y hasta más a varios programas. Para Cohen (2001), "el respaldo a la reforma de la educación superior y de las profesiones había aumentado durante los últimos años de manera constante en Colombia y en consecuencia un número reducido pero creciente de mujeres ingresaba a la universidad" (p. 211).

Hacia mediados de la década del sesenta el número de mujeres graduadas en las universidades colombianas había aumentado apreciablemente: de seis que obtuvieron sus títulos en 1938, a 232 en 1955 y 915 en 1965 (Cohen, 2001). Así mismo, es fundamental mencionar que por ese tiempo el ingreso de las colombianas fue transversal a dos momentos claves en la historia de la Universidad Nacional; por un lado, la Reforma Patiño, que constituyó la Ciudad Universitaria y otorgó posibilidades de vivienda y alimentación a las y los estudiantes que lo requerían a través de lo que se conoce como bienestar universitario; por otra parte, la centralización en el Estado, que implicó una nueva definición de la Universidad en la que se afirma como institución de carácter público que responde y atiende a las necesidades del país (Soto, 
2005). Por esta razón, el ingreso de la mujer a la Universidad Nacional de Colombia posibilitó la investigación en temas nuevos como los estudios sistemáticos de la familia, la niñez en la calle, la vejez y las violencias en el país en términos de sus implicaciones (Ramírez, 2010).

De otra parte, la realidad en la capital del Valle del Cauca no era muy distinta. En 1948 se graduó la primera promoción de la entonces denominada Universidad Industrial del Valle, en la ciudad de Santiago de Cali. El acta de grado inicial, tipeada en máquina de escribir, fue expedida por la institución el 15 de julio de 1948 y firmada por el rector Tulio Ramírez. En ese entonces fueron 25 mujeres las primeras egresadas que el alma máter graduó como enfermeras generales; de ellas no se tiene ninguna noticia, no hay archivos de matrícula o registros de calificaciones, solo un acta de grado que evidencia el importante papel que la mujer jugaba para entonces en los diferentes ámbitos de la vida académica y laboral de la universidad.

Después de esta primera promoción y por varios años los egresados de la universidad fueron médicos y químicos hombres, y mujeres enfermeras. Solo hasta 1958 uno de los programas académicos insignia de la institución en la época graduó a la primera arquitecta de la Universidad Industrial del Valle. En 1959 se graduó la primera mujer Doctor en Medicina y Cirugía y la primera ingeniera; en 1967 la primera bióloga, la primera psicóloga en 1968 y la primera economista en 1969 (Universidad del Valle, 2010).

\section{Mujeres terapeutas, hallazgos para la década 1960 - 1970}

Las funciones ejercidas por la mujer y el contexto de aquella época la determinaron como un ser sin capacidad de decisión, sujeto de instrucción y sacrificio. La Terapia Ocupacional empieza a construirse alrededor de ese contexto socio-histórico:

Al ser una carrera femenina pues, tiene relación directa en la visión de su ejercicio por la sociedad, con lo que la sociedad piensa que es el papel de la mujer en la misma. La TO cuando empieza alrededor del 70, empieza en un mundo en el que las mujeres no toman decisiones. Las decisiones las toman los varones. Solo en diciembre del año 71, y tras la aprobación de una ley, las mujeres toman decisiones de patria potestad. Hasta diciembre de 1974, solo en ese diciembre, la mujer no tuvo que pedirle permiso al marido para dar un permiso de viaje a los hijos, por ejemplo, una cosa tan simple. (C. H. Vergara, comunicación personal, Marzo de 2015).

Tras la caída del presidente Rojas Pinilla en 1957, las mujeres pueden elegir y son alrededor del 50\% de la población (Aguilera, 2003). Los contextos sociales y políticos que influyen en la creación de carreras para la mujer colombiana se relacionan con el logro del derecho a decidir, a tener voz y voto con el primer sufragio femenino, así como con las aperturas y cambios a nivel educativo antes mencionados. Así, los sesentas serían la década con mayor número de matrícula femenina en la Universidad Nacional, lo que debía generar transformaciones a nivel de programas académicos acordes a sus capacidades. 
El 1 de septiembre de 1959 la Facultad de Medicina de la Universidad Nacional de Colombia crearía el Servicio de Medicina Física y Rehabilitación, una unidad dependiente de la sección de Medicina Interna del Hospital San Juan de Dios de Bogotá que efectuaría actividades en los órdenes administrativo, docente y asistencial (Pardo, 1972). Ello dio origen en 1960 a la creación del curso para la educación posgraduada de médicos especialistas en Medicina Física y Rehabilitación.

Por la situación de violencia que venía atravesando el país se detectó una amplia demanda en la sección de rehabilitación, por lo que resultaba necesario constituir equipos completos de salud en dicha área (Escobar y Rodríguez, 2015). Por su parte los medios de comunicación se referían a la aparición de brotes de enfermedades infecto-contagiosas como la poliomielitis, tanto en la ciudad de Cali como en Bogotá. Las estadísticas de la época indican que "entre 1966 y 1980 se atendieron en el Instituto Colombiano de Ortopedia y Rehabilitación Franklin Delano Roosevelt de Bogotá 1.449 casos de poliomielitis aguda, de un total de 22.022 pacientes vistos en primera consulta" (Malagón \& Ávila, 1982).

Es así como desde el Hospital San Juan de Dios de Bogotá se hace la solicitud a la Facultad de Medicina de la Universidad Nacional para cubrir estas necesidades de formación. Por medio del Acuerdo 04 del 20 de enero de 1966, firmado por el Consejo Superior Universitario, se autoriza a la Facultad para que en la Sección de Rehabilitación se iniciaran labores de las tres carreras técnicas que vendrían a llenar el gran vacío existente en el campo de las ciencias de la salud: los programas de Terapia Física, Terapia de Lenguaje y Terapia Ocupacional, que tendrían una duración de seis semestres, compartirían el primer año y posteriormente recibirían clases propias de cada área técnica (Pardo, s.f). Mientras tanto, en Cali la jefa de sección de Enfermería del Hospital Universitario del Valle calificaba la situación de salud de la población como alarmante, y convocaba a la Universidad del Valle, por medio de la Facultad de Enfermería, a la instrucción de mujeres en el servicio y sacrificio en pro del cuidado de las personas afectadas (Taborda, 1970).

Un hallazgo que describe el origen y concepto de las terapeutas ocupacionales de la época es el Prospecto de la Carreras de Terapia (Figura 1), en el que se define que:
La terapeuta ocupacional tiene la obligación de planear y desarrollar un programa de actividades para cada pa- ciente, a fin de ayudar a su recupera- ción física o mental, de acuerdo a la orden del médico... debe ser afable y tener una personalidad bien equilibra- da... (Pardo, s.f.).

A diferencia de la Universidad Nacional de Colombia, para los sesentas y setentas la Universidad del Valle aún no abría su programa académico en Terapia Ocupacional. Sin embargo, algunos hechos relevantes ya demostraban la importancia de la participación de la mujer en el sector salud, en la enseñanza y en procesos de liderazgo, así como la percepción de sí en aquella época. Un enunciado identificado en la sección Femeninas del periódico 
Figura 1. Prospecto Carreras de Terapia. En el cual se dan las primeras definiciones de la Terapia Ocupacional y del perfil de las terapeutas.

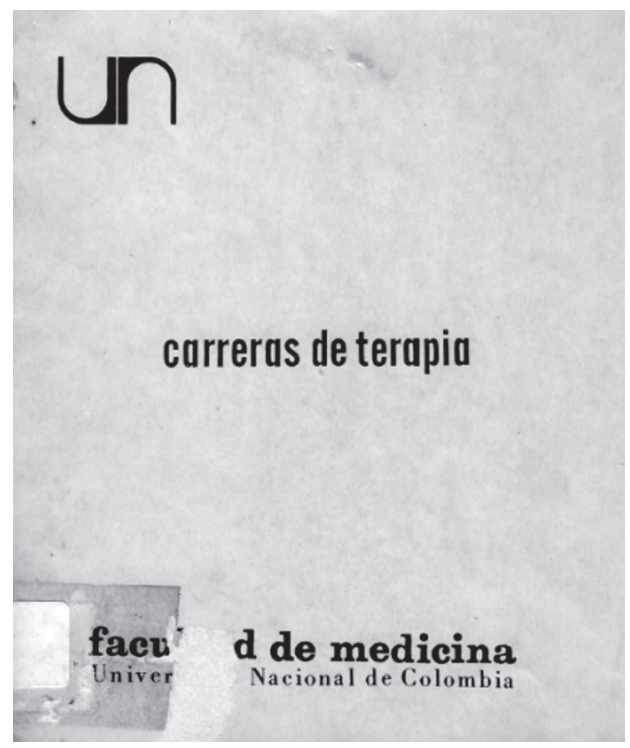

Fuente: Fotografía del original escrito por Jorge Pardo Ruiz, (s.f).

El País de 1971 expone la importancia de la participación de la mujer en profesiones femeninas como Enfermería; así mismo, invoca el trabajo de la mujer para las labores de cuidado de personas enfermas que requieren de mucha asistencia, esto a manera de voluntariado (Figura 2), es decir sin remuneración, indicando que el Hospital Universitario afrontaba una crisis económica debido a los distintos frentes que debía atender con los pacientes: medicamentos, aparatos ortopédicos y rebajas en los costos de hospitalización; además contaban con escasas voluntarias y aspiraban a que las señoras, tomando conciencia de lo que era el servicio a los semejantes, se animaran a ingresar al voluntariado (Guevara, 1971).
Figura 2. El voluntario hospitalario necesita más colaboradoras.

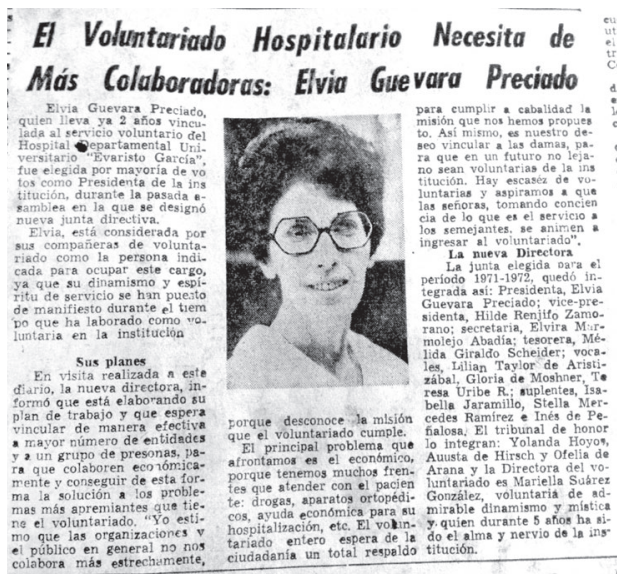

Fuente: Fotografia tomada del original publicado en el diario El País (p. 17) el 14 de febrero de 1971.

Como ocurría en la creación de la Terapia Ocupacional en Argentina:

La cual estuvo atravesada por un sistema de género que establece jerarquías, privilegios y desigualdades delimitando un campo profesional ligado a un mundo considerado como naturalmente femenino constituido por las actividades de la vida diaria, las actividades artesanales y manuales, el cuidado de los niños y una cuota de amor y abnegación para llevar adelante el desafío de la rehabilitación (Testa \& Spampinato, 2010, pág. 178).

De manera similar se originaron algunas profesiones en Colombia, como la Terapia Ocupacional; se enmarcaban en la mujer unas habilidades con las que pudiera guiar a las personas en sus relaciones diarias, saber reconocer sus logros y estimularlos cuando fuera necesario. Lo anterior permite identificar una serie de funciones que debía ejercer, entre ellas: prestar servicios, dar medicamentos, proporcio- 
nar aparatos ortopédicos, cumplir con prescripciones médicas, prestar atención individual, guiar en las relaciones diarias, reconocer los logros del otro, cuidar a personas afectadas, hacer cosas por los demás, llevar a cabo funciones manuales y artesanales, enseñar y adaptar a los niños y resolver sus necesidades particulares. Se atribuye a las mujeres la entrega total a los demás, el autocontrol de las emociones y la suavidad; cualidades de sacrificio similares a las virtudes de la vida religiosa y familiar, es decir, del ámbito de lo privado.

La creación de programas académicos de Terapia Ocupacional en la Universidad Nacional de Colombia y posteriormente en la Universidad del Valle fue impulsada y motivada principalmente por hombres médicos que tenían un interés marcado por el tema de la rehabilitación. Esta situación permaneció con el paso del tiempo, el médico hombre tuvo total influencia sobre las decisiones que las terapeutas ocupacionales debían tomar, situación reconocida por terapeutas de la época: "...entonces cuando ustedes ven una profesión femenina y una profesión paramédica en el año 70, es porque todo se hacía bajo prescripción médica: la mujer no puede prescribir porque no podía tomar decisiones que carguen con responsabilidad" (C. H. Vergara, comunicación personal, marzo de 2015).

Pese a estos roles asignados por una sociedad conservadora, industrial y patriarcal, las mujeres también asumieron durante la década de los setenta un papel de liderazgo en la movilización de sujetos para la creación de los juegos en silla de ruedas, la conformación de agremiaciones de personas con discapacidad, el impulso de nuevos campos en instituciones de rehabilitación, entre otras situaciones y oportunidades para la población con discapacidad de aquella época. Así lo refiere la terapeuta ocupacional Carmen Helena Vergara, en otro de sus relatos:

En 1970, en el mes de enero, se hicieron los juegos panamericanos en silla de ruedas en la ciudad de Buenos Aires. Allí se encontraba un médico deportólogo de apellidos Torres Baldó, y con él trabajaba una mujer llamada Valery Tausend, fisioterapeuta. Ella movilizó en este país mil cosas que la gente ya no recuerda. Una de ellas fueron los juegos en silla de ruedas, asesorando a la Asociación Colombiana de Parapléjicos - ASCOPAR, y se impulsó la creación de una delegación principalmente conformada por su gente para ir como observadores a los Juegos Panamericanos, y de allí salieron los paralímpicos en Colombia, de esa delegación que fueron en enero de 1970 a los juegos. (C. H. Vergara, comunicación personal, 15 de 2015).

Además, se destaca el papel de distintas terapeutas ocupacionales que durante la década de los setentas impulsaron la creación de servicios de Terapia Ocupacional en instituciones del Estado como el Hospital Universitario del Valle y el Instituto Colombiano de Seguros Sociales. También el de aqueIlas que crearon en Santiago de Cali los centros de atención diurna en el campo de rehabilitación psicosocial, y de quienes abrieron el campo educativo para la integración escolar a través de su participación en la Secretaría de 
Educación (C. H. Vergara, comunicación personal, 15 de 2015).

Todo lo anterior demarca el contexto en el cual nace la Terapia Ocupacional en Colombia, puntualmente en las ciudades de Cali y Bogotá. Se empieza a construir una carrera paramédica al servicio del cuidado de las personas, junto con otras profesiones como la Psicología, la Enfermería, la Fonoaudiología, la Fisioterapia y la Pedagogía. La participación de la mujer en aquella época inscribe una pauta en el desarrollo de profesiones para el cuidado de la comunidad; así mismo, la mujer es un sujeto importante que lucha contra el estigma de una sociedad en donde la capacidad de decisión estaba determinada por condiciones de patria potestad y género.

\section{Reflexiones y conclusiones contrastadas}

Como se describe previamente, la Terapia Ocupacional parece haberse iniciado como programa de formación alrededor de un contexto sociohistórico en el cual la mujer adquirió el derecho de participar en la toma de decisiones políticas, económicas, educativas y sociales, libertad que aparece luego de la legitimación del derecho al voto femenino. Sin embargo, los acontecimientos aquí socializados hacen referencia al papel de la mujer como un ser sin capacidad de decisión, sujeto de instrucción y sacrificio, por lo cual, su derecho se ejercía mediado por su desempeño en profesiones subordinadas, paramédicas, de cuidado del otro. La formación de terapeutas ocupacionales en el país, como se ha descrito, fue en sus inicios prefe- rentemente femenina y orientada al cuidado de otros. Con el paso de los años esta realidad se transformó con el empoderamiento de aquellas mujeres por alcanzar una formación de mayor nivel y con capacidades de participación.

De otro lado, la entrada de las mujeres a la universidad entre los sesentas y setentas, y la consecuente conquista de un título universitario potenciaron, sin lugar a dudas, su autonomía y su libertad de pensamiento (Soto, 2005). Esto se hace evidente al conocer que la creación de los programas académicos de Terapia Ocupacional, tanto en la Universidad Nacional como en la Universidad del Valle, fueron impulsadas por dos médicos, Jorge Pardo y Jaime Villaquirán, respectivamente, mientras las terapeutas ocupacionales se encontraban bajo su dirección. Esa situación se transformaría más adelante con las reformas de plan curricular, por ejemplo, en el paso de la formación técnica a la licenciatura en la Universidad Nacional de Colombia en 1973 (Escobar \& Rodríguez, 2015).

Este ejercicio reflexivo y las investigaciones que lo provocaron abren una perspectiva distinta para analizar el origen de la Terapia Ocupacional en el país, semejante a las adoptadas en Argentina, Chile o Brasil. En ellas se avanza en la comprensión de la profesión a partir de los contextos socios históricos mencionados y la aproximación a historias de vida de mujeres que iniciaron esta lucha. Aunque las mujeres de la época no enfrentaran las estructuras de poder existentes para trabajar explícitamente por la equidad de género, sí lograron utilizar efectiva- 
mente los estereotipos que como mujeres se les imponían para plantear sus intereses y exigencias en la formación profesional. Fue así como se abrieron campo en el mundo laboral, manteniendo el reconocimiento social como cuidadoras del hogar y de los otros, hasta lograr una formación adecuada en áreas relacionadas con la solidaridad y la asistencia social, pero que socialmente continuaban siendo aceptadas como adecuadas para mujeres; utilizaron el rol de madres para crear la necesidad de recibir la educación y el acompañamiento apropiados para desempeñarlo.

Así pues, la Terapia Ocupacional se constituye en una oportunidad que tiene la mujer para declarar su autonomía como ser humano y desarrollar su capacidad de contribuir a la sociedad de una forma distinta a la declarada como don natural. Cabe resaltar que la Terapia Ocupacional colombiana ha cambiado, que en la actualidad muchas terapeutas ocupacionales han encaminado su ejercicio a campos que antes se consideraban masculinos, y que también hay un número creciente de hombres interesados en la Terapia Ocupacional, con aspiraciones profesionales diversas.

Finalmente, hacemos un reconocimiento a las mujeres, en particular a las terapeutas ocupacionales de las décadas de los sesenta y setenta, quienes marcaron un punto de partida fundamental para el desarrollo de la profesión, asumiendo de forma valerosa las decisiones respecto a la construcción de la profesión y la conformación de un hogar. Esta también es una invitación a participar en la lectura y el aná- lisis histórico del rol y la participación de mujeres y hombres en las transformaciones, luchas y resistencias en cualquier contexto. Estos constituirán aportes a la Terapia Ocupacional para desarrollar en profundidad la proyección de las y los futuros profesionales.

\section{Agradecimientos}

A Jaqueline Cruz Perdomo, Clara Duarte Cuervo, Aleida Fernández Moreno y Solángel García Ruiz.

\section{Referencias}

Aguilera, M. (2003). Por primera vez, la mujer tuvo derecho a votar en 1853, 150 años de la Constitución de la provincia de Vélez. Revista Credencial Historia, (163), Recuperado de http://admin.banrepcultural.org/node/74727

Cohen, L. (2001). Colombianas a la vanguardia. Medellín: Universidad de Antioquia.

Correa, M. (1947, Abril). Contestación a la encuesta. Revista Letras y Encajes Semana, (249), 128. Recuperado de http:// www.bdigital.unal.edu.co/50196/1/letrasyencajesabril1947.pdf

Escobar, X., \& Rodríguez, L. (2015). Terapia Ocupacional: Una perspectiva histórica desde la Universidad Nacional de Colombia (1966-1989). (Trabajo de grado). Bogotá: Universidad Nacional de Colombia.

Guevara, E. (1971, 14 de Febrero). El voluntario hospitalario necesita mas colaboradoras. El País, p. 17.

Herrera, M. (1993). Historia de la educación en Colombia. La República Liberal y la modernización de la educación: 19301946. Recuperado de http://www.pedagogica.edu.co/storage/rce/articulos/ rce26_06ensa.pdf

López, R. (2002). La universidad femenina, la ideología de género y el acceso de las 
Colombianas a la educación superior 1940 - 1958. Revista Historia de la Educación Latinoamericana, (4), 67-90.

Malagón, V. , \& Ávila, G. (1982). Resumen de un simposio sobre parálisis infantil presentado en la Academia Nacional de Medicina en mayo de 1982. Carta Ortopédica, 6 (34). Recuperado de https://encolombia. $\mathrm{com} / \mathrm{medicina/revistas-medicas/ortope-}$ dia/vo-121/orto12198estado/

Melgarejo, M. (2000). El pensar histórico como genealogía: acto interpretativo y construcción de subjetividad. Revista Fronteras de la Historia, 5, 35-50.

Munevar, D. (2015, 11 de Marzo). Historias de Puertas para Adentro no contadas en el Hospital San Juan de Dios. Apuntes de línea de profundización. Bogotá: Universidad Nacional de Colombia.

Palma, D., \& Gómez, L. (2015). Los inicios de la terapia ocupacional en Santiago de Cali: Relatos de un ejercicio (Proyecto de profundización de la Universidad del Valle). Cali, Colombia: Universidad del valle.

Pardo, J. (1972). Curso para la Formación de Médicos Especialistas en Rehabilitación. Bogotá: Universidad Nacional de Colombia.

Pardo, J. (s.f). Carreras de Terapia. Bogotá: Facultad de Medicina, Universidad Nacional de Colombia.
Ramírez, M. (2010, 8 de marzo). La Mujer en la Historia de la Educación Superior en Colombia. Recuperado de http://noticias.universia.net.co/vida-universitaria/ noticia/2010/03/08/258244/mujer-historia-educacion-superior-colombia.html

Soto, D. (2005). Aproximación Histórica a la Universidad Nacional de Colombia. Revista Historia de la Educación Latinoamericana, (7), 99 - 136.

Subgerencia Cultural del Banco de la República. (2015). El voto femenino. Recuperado de: http://www.banrepcultural. org/blaavirtual/ayudadetareas/politica/ el_voto_femenino

Taborda, L.T (1970, 12 de Mayo). Es alarmante el aumento de Parálisis Infantil en la ciudad. El país, Sección Femeninas, p. 12.

Testa, D. E., \& Spampinato, S. B. (2010). Género, salud mental y terapia ocupacional: algunas reflexiones sobre la influencia de la historia de las mujeres y la perspectiva de género en nuestras prácticas. Revista de Terapia Ocupacional da Universidade de São Paulo, 21(2), 174-181.

Universidad del Valle. (2010). Las primeras egresadas de la universidad. Revista Campus (87). Recuperado de: http://campus. univalle.edu.co/2010/marzo/edicion87/ egresadas.php 